\title{
Early pulmonary rehabilitation after acute exacerbation of COPD: a randomised controlled trial
}

\author{
Jakob L. Kjærgaard ${ }^{1}$, Carsten B. Juht ${ }^{2,3}$, Peter Lange $e^{1,4}$ and Jon T. Wilcke ${ }^{1}$
}

Affiliations: 'Dept of Internal Medicine, Herlev and Gentofte Hospitals, University of Copenhagen, Copenhagen, Denmark. ${ }^{2}$ Dept of Physiotherapy and Occupational Therapy, Herlev and Gentofte Hospitals, University of Copenhagen, Copenhagen, Denmark. ${ }^{3}$ Research Unit for Musculoskeletal Function and Physiotherapy, Dept of Sports Science and Clinical Biomechanics, University of Southern Denmark, Odense, Denmark. "Section of Epidemiology, Institute of Public Health, University of Copenhagen, Copenhagen, Denmark.

Correspondence: Jakob L. Kjærgaard, Dept of Internal Medicine, Herlev and Gentofte Hospitals, University of Copenhagen, Gentofte Hospitalsvej 1, DK-2900, Denmark. E-mail: jakobanaj.dk

\section{ABSTRACT}

Objectives: The aim of this study was to establish whether early pulmonary rehabilitation after severe exacerbation of chronic obstructive pulmonary disease (COPD) reduces mortality and hospital admissions, and increases physical performance and quality of life compared to rehabilitation initiated later in the stable phase of COPD.

Methods: In a randomised controlled trial of 150 patients hospitalised with an exacerbation of COPD, participants were allocated to pulmonary rehabilitation either within 2 weeks after discharge or the same rehabilitation programme but initiated 2 months after discharge.

Results: Early pulmonary rehabilitation did not prolong time to first hospital admission or time to death (hazard ratio $0.79,95 \%$ CI $0.47-1.23, \mathrm{p}=0.33$ ) compared to rehabilitation in stable phase. However, 2 months after inclusion, pulmonary rehabilitation resulted in a significantly better improvement in the incremental shuttle walk test $(33.9 \mathrm{~m}, 95 \%$ CI $4.18-63.7, \mathrm{p}=0.02)$ compared to that in the stable phase. The difference in the endurance shuttle walk test was of borderline significance (140 s, 95\% CI -2.03-282.76, $\mathrm{p}=0.05$ ), but there was no significant difference concerning the COPD assessment test $(-1.43$ points, $95 \%$ CI $-3.44-0.59, \mathrm{p}=0.17)$.

Conclusion: Early pulmonary rehabilitation after acute exacerbation of COPD led to a faster improvement in physical performance compared to rehabilitation initiated later in the stable phase, but did not improve survival or prolong time to hospital readmission.

@ERSpublications

Early pulmonary rehabilitation shortly after \#AECOPD leads to a faster recovery in physical performance than initiated in the stable phase. Adherence to rehabilitation is significantly higher when initiated shortly after an exacerbation. http://bit.ly/2S7XsT9

Cite this article as: Kjærgaard JL, Juhl CB, Lange P, et al. Early pulmonary rehabilitation after acute exacerbation of COPD: a randomised controlled trial. ERJ Open Res 2020; 6: 00173-2019 [https:// doi.org/10.1183/23120541.00173-2019].

This article has supplementary material available from openres.ersjournals.com

This study is registered at www.clinicaltrials.gov with identifier number NCT02987439. The study group has a long history of data sharing. The data used for the current study will be made available to other researchers. However, the study group has the obligation to assure that data shared are handled in a way that complies with the science ethics permission and the General Data Protection Regulation. Apart from that, the study group has an ambition that data shared are used for sound scientific purposes by researchers. We encourage researchers who find interest in sharing our data, to contact the corresponding author. The corresponding author will then bring this request to the study group. Data will be shared if the abovementioned issues are assessed as compliant.

Received: 11 July 2019 | Accepted after revision: 11 Dec 2019

Copyright $\odot$ ERS 2020. This article is open access and distributed under the terms of the Creative Commons Attribution Non-Commercial Licence 4.0. 


\section{Introduction}

Acute exacerbation of chronic obstructive pulmonary disease (AECOPD) is frequent in severe chronic obstructive pulmonary disease (COPD). AECOPD is among the most common causes of hospitalisation, it increases the decline in lung function and repeated exacerbations are associated with higher readmission rates to hospital and increased mortality [1-3]. Furthermore, severe AECOPD is associated with worsening of symptoms and impairment of physical and psychological wellbeing [4,5]. Although symptoms after AECOPD usually last 7-10 days, about $20 \%$ of the patients have not fully recovered after 8 weeks [6].

Pulmonary rehabilitation plays a key role in the management of COPD. It has a significant impact on reducing symptoms and increasing exercise capacity and quality of life in patients with stable COPD [7]. Due to the deterioration in the patient's physical performance and quality of life during exacerbation, pulmonary rehabilitation initiated immediately after AECOPD is expected to show a larger beneficial effect compared to when it is introduced later in stable COPD. In fact, early pulmonary rehabilitation after AECOPD seems to reduce the risk of recurrent hospital admissions and mortality, although the results have not been uniform, and one large study reported increased mortality [8, 9]. However, in spite of great heterogeneity between the studies, a systematic review by PuHAN et al. [10] in 2016 evaluating the effect of early rehabilitation compared to no rehabilitation, concluded that pulmonary rehabilitation is an effective intervention after an exacerbation regarding exercise capacity and health-related quality of life and also found beneficial effects on mortality and hospital admissions. The investigators suggested that the heterogeneity between the results could be explained by the difference in the extensiveness of the rehabilitation programmes and the methodological quality of the studies.

We therefore conducted a randomised controlled trial investigating the effect of a comprehensive and standardised pulmonary rehabilitation programme initiated within 2 weeks after discharge from hospital compared to a similar rehabilitation programme initiated in the stable phase, 2-3 months after discharge, in patients admitted with an AECOPD.

\section{Methods}

Study design

This study was an investigator-initiated single centre open-label randomised controlled clinical trial conducted at the Department of Respiratory Medicine at Gentofte Hospital in Denmark. Approval was obtained from the local ethics committee (H-2-2013-143) and the study was registered in ClinicalTrials.gov (NCT02987439). We used sealedenvelope.com to prepare the sequence allocation and the patients were allocated 1:1 to either early or later pulmonary rehabilitation, using sealed opaque envelopes. Participants readmitted to hospital during the study were not randomised again but remained in their initial treatment group.

We assessed data at discharge from hospital at baseline and outcomes at 2 and 6 months follow-up. Mortality and hospital readmissions were recorded at 12 months.

\section{Primary outcome}

Composite outcome of 1-year mortality was measured as all-cause mortality at 12 months and readmission was defined as at least one hospital readmission due to any cause in the 12-month follow-up period. This outcome was chosen as it was used in a Cochrane review in 2011 [11].

\section{Secondary outcome}

These included: 1-year all-cause mortality and time to death, all-cause hospital readmission and time to readmission, proportion of participants with at least one hospital readmission due to any cause, hospital readmission due to a respiratory cause, exercise performance measured by the incremental shuttle walk test (ISWT) [12] and the endurance shuttle walk test (ESWT) [13], and health-related quality of life measured by the COPD assessment test (CAT) [14].

\section{General care}

During hospitalisation, the participants in both groups were given standard medical treatment for AECOPD, optimised prescribed medical treatment of COPD and were instructed in chest physiotherapy [15]. At 3 to 7 days after discharge, baseline spirometry (pulmonary function test (PFT)), Medical Research Council (MRC) dyspnoea scale, CAT, smoking status, ISWT and ESWT were recorded. Information on smoking cessation was given if relevant. Participants were introduced to a telephone hotline operated by a COPD nurse during office hours, where they could seek advice about their COPD. At 2 and 6 months after discharge, smoking status, MRC, CAT, PFT, ISWT and ESWT were recorded. 


\section{Early pulmonary rehabilitation group}

After discharge, the participants were referred, according to their physical status, to either outpatient rehabilitation in the hospital or in the community or rehabilitation at home. Rehabilitation was initiated within 2 weeks after discharge.

The programme was a standardised 7-week programme with supervised training sessions, consisting of aerobic exercise and strength training twice weekly for $1.5 \mathrm{~h}$ and educational session once per week for $1 \mathrm{~h}$. The programme fulfilled the criteria for pulmonary rehabilitation, according to the Danish Health Authority and the British Thoracic Society guidelines [16, 17]. The detailed description of the rehabilitation programme is provided in the supplementary material.

\section{Stable pulmonary rehabilitation group}

At the 2-month follow-up, participants in the stable pulmonary rehabilitation group were referred to a similar programme as undertaken by the early pulmonary rehabilitation group.

\section{Study population}

Eligible patients were included consecutively between 2013 and 2016 at the Department of Respiratory Medicine, Gentofte Hospital. Inclusion criteria were hospitalisation with an AECOPD, a diagnosis of COPD according to the Global Initiative for Chronic Obstructive Lung Disease (GOLD) [18], age $>18$ years, ability to walk $10 \mathrm{~m}$ independently (with or without a walking aid). Exclusion criteria were: life expectancy $<6$ months due to another disease (such as cancer or severe heart disease), difficulties in understanding and speaking Danish (e.g. due to dementia), residence outside Gentofte Hospital's recruitment area, discharge to a residence other than their own (e.g. nursing home), participation in another rehabilitation programme or inability to provide informed consent.

Figure 1 shows a flowchart with the description of the study design.

\section{Statistical analysis}

For the sample size calculation, we estimated 1-year mortality in patients without previous hospital admissions to be $15 \%$, the expected reduction in mortality or readmission rate was set at $25 \%$ (power $80 \%$ and $\alpha=0.05$ ) leading to a requirement of 112 participants (56 in each group). The effect size was estimated from the results of the Cochrane review in 2011 [11]. With an estimated dropout frequency of $20 \%$, we recruited 150 participants. We used STATA 13 (STATA Corporation, College Station, Texas, USA) for the statistical analyses and analyses of the primary and secondary outcomes were performed according to the intention-to-treat principle.

Baseline characteristics were analysed with a Student's t-test or Mann-Whitney U-test for continuous data and Chi-squared or Fisher's exact test for categorical data. The primary outcome was analysed with Fine and Gray competing risk analysis with readmission as failure and death as competing risk. For secondary outcomes, we used logistic regression for mortality ratio and readmission ratio, log-rank test and Cox proportional hazards model for survival analysis, and negative binomial regression for relative risk of readmission between groups. Odds ratios (ORs), hazard ratios (HRs) and incidence rate ratios (IRRs) are shown with the control group as reference. Longitudinal data were analysed with a mixed-effects model for CAT, ISWT and ESWT, assuming values were missing at random.

\section{Results}

\section{Baseline}

We screened 697 patients hospitalised with AECOPD over a 4-year period. We included 150 participants, with 76 in the early pulmonary rehabilitation group and 74 in the stable pulmonary rehabilitation group. The main reasons for non-inclusion were: declining to participate, estimated short life expectancy and participation in other rehabilitation programmes. Figure 2 shows a flow diagram of the progress throughout the study. One participant was randomised twice by mistake. During the 6-month follow-up period, 6 and 13 participants were excluded in the early and stable pulmonary rehabilitation groups respectively, mainly due to a diagnosis of asthma or start of another rehabilitation programme. This resulted in 70 and 61 participants in the early and stable pulmonary rehabilitation groups, respectively and all were included in the analysis of the primary end-point.

Baseline characteristics of the participants are presented in table 1 and showed a generally well-balanced randomisation and they resembles the general COPD population hospitalised with AECOPD in Denmark [19]. However, participants in the stable pulmonary rehabilitation group had a better CAT score. 
FIGURE 1 Flowchart of the study design. COPD: chronic obstructive pulmonary disease; PR: pulmonary rehabilitation: AECOPD: acute exacerbation of COPD; GOLD: Global Initiative for Chronic Obstructive Lung Disease; PFT: pulmonary function test: MRC: medical research council dyspnoea scale; ISWT: incremental shuttle walk test; ESWT: endurance shuttle walk test; CAT: COPD assessment test.

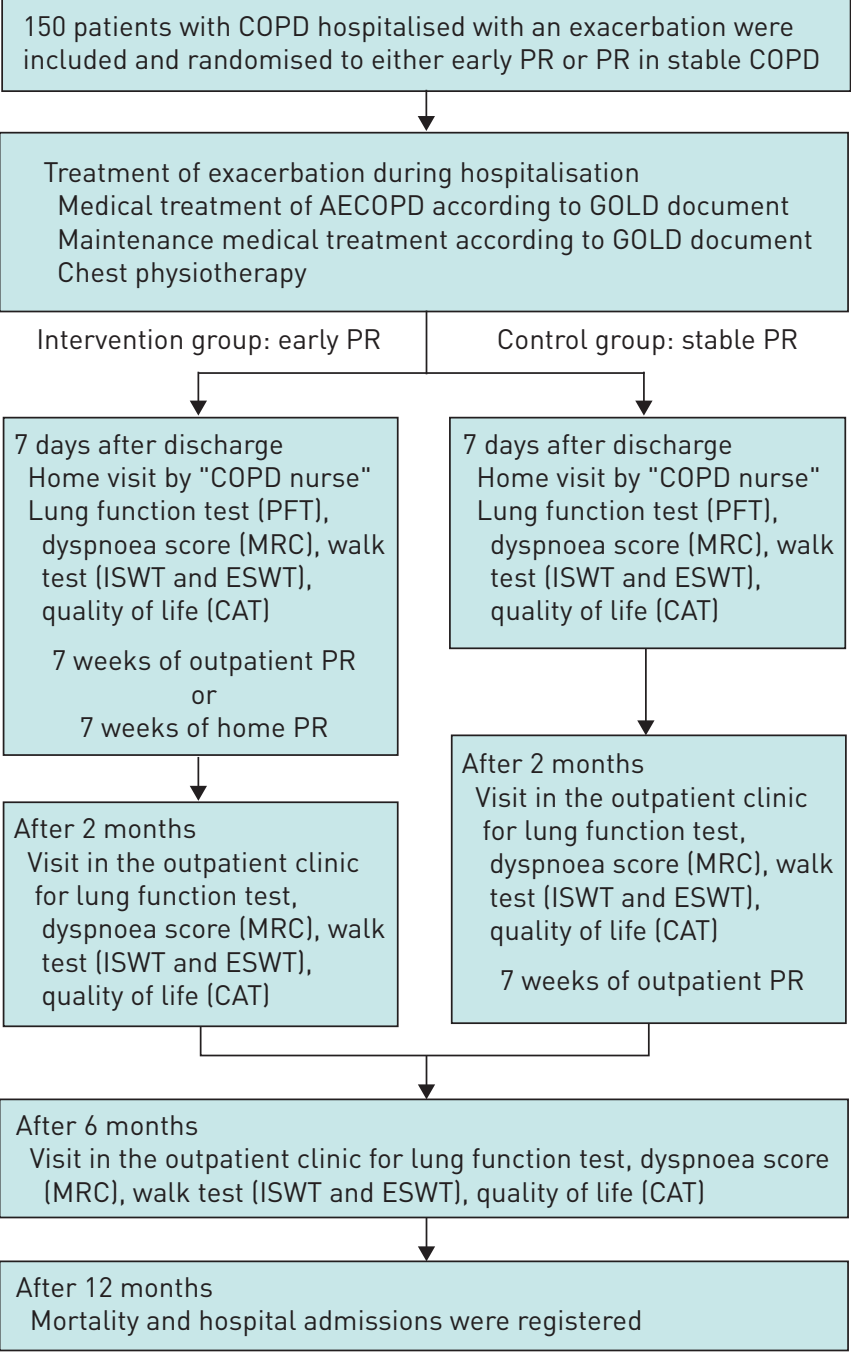

\section{Adherence}

In the early pulmonary rehabilitation group, 58 (83\%) participants attended at least one rehabilitation session and 39 (56\%) met our a priori definition of adherence (attendance $>75 \%$ of sessions). The median time to the first session from discharge was 9 days (interquartile range (IQR) 5-15) with a median number of sessions of 13 (IQR 8-16). Forty-seven participants received rehabilitation at the hospital's facilities and five in the community. Six participants were initially trained in their own homes, but only for a few sessions and hereafter to complete the programme in the outpatient setup. In the stable pulmonary rehabilitation group, $32(53 \%)$ had at least one rehabilitation session within 1 year after inclusion with a median number of sessions of 13 (IQR 10-16) and 21 (34\%) participants were adherent. The participants in the early pulmonary rehabilitation group were significantly more adherent (OR 2.39, 95\% CI 1.11-5.18, $\mathrm{p}=0.01$ ).

\section{Primary outcome}

The primary outcome was a composite end-point of hospitalisation or death. In total, 78 participants (42 in the early and 36 in the stable pulmonary rehabilitation group) met this end-point. Except for one patient who died without previous hospital admission, all were readmitted at least once in the 12-month follow-up period. In the competing risk analysis, we found a crude nonsignificant risk (HR 1.03, 95\% CI $0.66-1.60, \mathrm{p}=0.91)$ and when adjusted for age, sex, forced expiratory volume in $1 \mathrm{~s}\left(\mathrm{FEV}_{1}\right)$, CAT and ESWT, there was a nonsignificant risk reduction (HR 0.79, 95\% CI 0.47-1.23, $\mathrm{p}=0.33$ ) (figure 3 ).

\section{Mortality}

Out of the 131 participants, 15 died during the study period, resulting in a 1-year-mortality rate of $11 \%$. One person was hospitalised and died before the baseline visit, five died of COPD, four of cancer, three of 


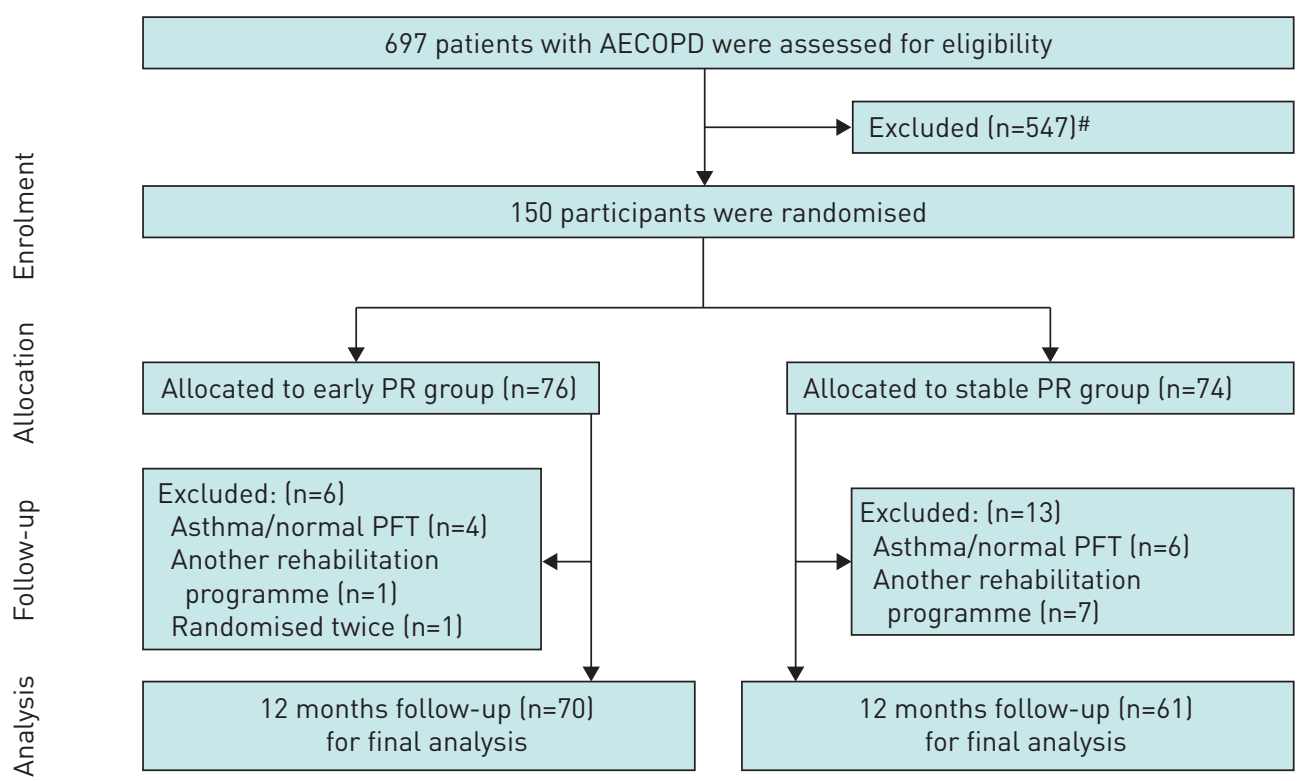

FIGURE 2 Screening, randomisation and study completion. AECOPD: acute exacerbation of chronic obstructive pulmonary disease; PR: pulmonary rehabilitation; PFT: pulmonary function test. ${ }^{\#}$ : excluded ( $\left.n=547\right)$ : declined to participate $(n=125)$; death in hospital $(n=30)$; life expectancy $<6$ months $(n=134)$; other rehabilitation programme $(n=126)$; living in nursing home $(n=29)$; not COPD $(n=30)$; residence outside Gentofte Hospital's admission area $(n=73)$.

cardiac causes, one of urosepsis and one of unknown cause. Nine participants died in the early pulmonary rehabilitation group (13\%), and six (10\%) in the stable pulmonary rehabilitation group. There were no significant differences between the groups with an adjusted OR of 1.05 (95\% CI 0.33-3.40) $\mathrm{p}=0.92$. A Kaplan-Meier plot of time to death is shown in figure 4 (log-rank test $\mathrm{p}=0.59$ ) and using the Cox proportional hazard model, the adjusted HR was 1.04 (95\% CI 0.36-3.01) $\mathrm{p}=0.95$.

\section{TABLE 1 Baseline characteristics}

\section{Characteristics}

\section{Age years \\ Male $n$ (\%)}

FEV $_{1}$ L

FEV $1 \%$ predicted

MRC dyspnoea score

CAT score

LTOT $\mathbf{n}(\%)$

BMI $\mathrm{kg} \cdot \mathrm{m}^{-2}$

Smoking $n(\%)$

Current

Former

Never

Exposure pack-years

GOLD assessment

Group C n (\%)

Group D n (\%)

ISWT distance $m$

ESWT time $s$

ESWT level
Early PR group $(n=70)$

Stable PR group $(n=61)$
$74.4 \pm 7.8$

$25(41)$

$1.0 \pm 0.4$

$44.1 \pm 17$

$3.1 \pm 0.8$

$14.7 \pm 5.3$

5 (8)

$24.2 \pm 4.1$

15 (25)

$43(70)$

3 (5)

$43.5 \pm 19.5$

$4(6)$

5 (8)

56 (92)

$188 \pm 110$

$263 \pm 210$

$6.3 \pm 3.6$

Data are presented as mean \pm SD unless otherwise stated. PR: pulmonary rehabilitation; FEV ${ }_{1}$ : forced expiratory volume in $1 \mathrm{~s}$; MRC: Medical Research Council; CAT: COPD Assessment Test; LTOT: long-term oxygen therapy; BMI: body mass index; GOLD: Global Initiative for Chronic Obstructive Lung Disease; ISWT: incremental shuttle walk test; ESWT: endurance shuttle walk test. 
FIGURE 3 Cumulative incidence of hospital readmissions and mortality. Competing risk analysis (Fine and Grayl of hospital readmission with death as competing risk. Adjusted for age, sex, forced expiratory volume in $1 \mathrm{~s}$ and COPD Assessment Test. Hazard ratio 0.79 (95\% Cl 0.47-1.23), $p=0.33$. Arrows show the start of pulmonary rehabilitation (PR).

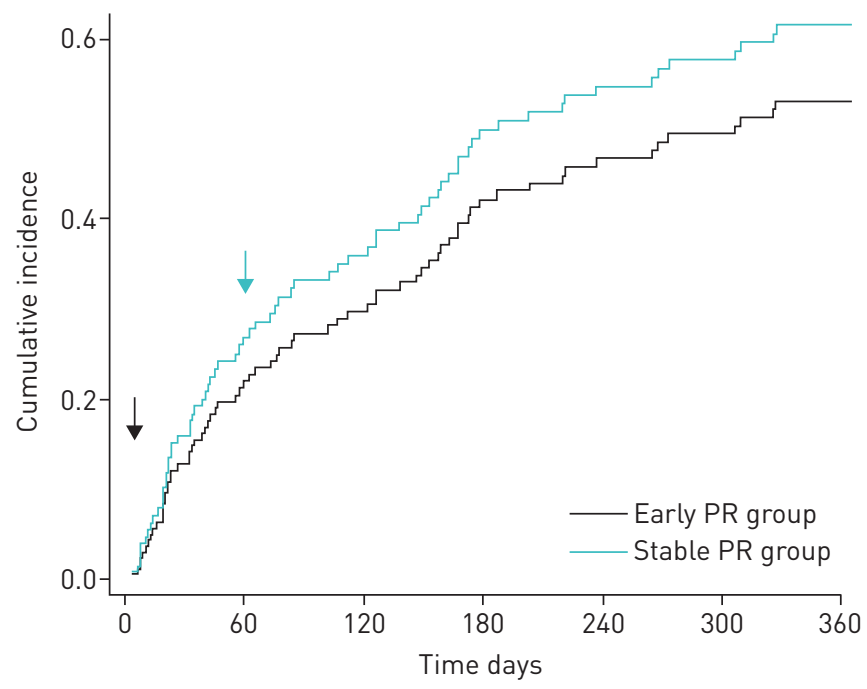

\section{Hospital admissions}

During the 1-year follow-up period, 77 (59\%) participants had a total of 218 readmissions due to any cause. In the early pulmonary rehabilitation group, 41 (59\%) participants had 128 readmissions versus 36 $(60 \%)$ in the stable pulmonary rehabilitation group having 90 readmissions. No significant reductions were found in participants with at least one readmission in the early pulmonary rehabilitation group, showing an adjusted OR of 0.57 ( $95 \%$ CI $0.25-1.31$ ) p=0.19 and we observed no significant difference in the time to first hospitalisation, with an adjusted HR of 0.76 (95\% CI $0.47-1.24) \mathrm{p}=0.28$. The number of admissions was also similar (IRR $1.04(95 \%$ CI $0.66-1.65) \mathrm{p}=0.85)$ in the two groups.

Sixty-two (47\%) participants had 151 admissions (86 in the early, versus 65 in the stable, pulmonary rehabilitation group) due to a respiratory cause (e.g. AECOPD or pneumonia). We found no significant difference between the groups in participants with at least one respiratory readmission (adjusted OR of $0.66(95 \%$ CI $0.27-1.37) \mathrm{p}=0.31$ ) and no difference in the time to respiratory readmission (adjusted HR of 0.78 (95\% CI $0.43-1.24) \mathrm{p}=0.35)$.

All analyses on mortality and readmission were adjusted for age, sex and baseline $\mathrm{FEV}_{1}$, CAT and ESWT and no interactions were found.

\section{Exercise performance and quality of life}

For physical performance and quality of life, there was significant improvement in both groups from baseline at 2 and 6 months (table 2). Although no statistical difference between groups was found at 6 months, the early pulmonary rehabilitation group gained $72 \mathrm{~m}$ in the ISWT at 2 months compared to the

FIGURE 4 Mortality. Kaplan-Meier plot showing survival. Log-rank test $p=0.59$. Arrows show the start of pulmonary rehabilitation (PR).

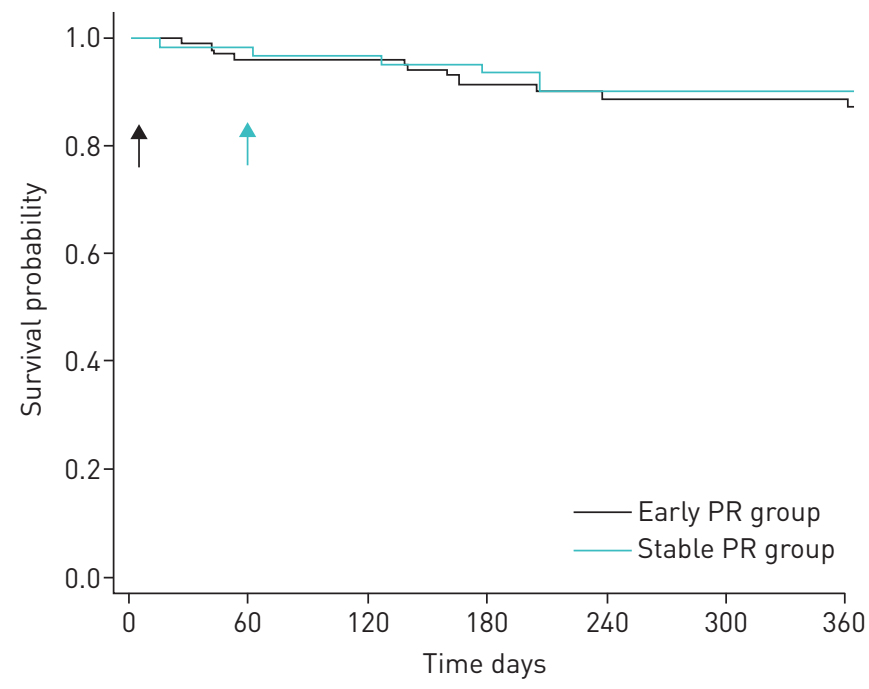




\begin{tabular}{|c|c|c|c|c|}
\hline & $\begin{array}{l}\text { Early PR group } \\
\text { change from baseline }\end{array}$ & $\begin{array}{l}\text { Stable PR group } \\
\text { change from baseline }\end{array}$ & $\begin{array}{l}\text { Difference between } \\
\text { groups }(95 \% \mathrm{CI})\end{array}$ & p-value \\
\hline \multicolumn{5}{|c|}{$\begin{array}{l}\text { Incremental shuttle } \\
\text { walk test } \mathrm{m}\end{array}$} \\
\hline Baseline & $193.3 \pm 8.8$ & $192.7 \pm 9.0$ & & \\
\hline 2 months & $72.4 \pm 9.5^{* * *}$ & $38.5 \pm 9.5^{* * *}$ & $33.9(4.2-63.7)$ & 0.02 \\
\hline 6 months & $68.1 \pm 10.0 * * *$ & $50.4 \pm 10.0 * * *$ & $17.7(-13.3-48.7)$ & 0.26 \\
\hline \multicolumn{5}{|c|}{$\begin{array}{l}\text { Endurance shuttle } \\
\text { walk test s }\end{array}$} \\
\hline Baseline & $239 \pm 42.4$ & $253 \pm 44.1$ & & \\
\hline 2 months & $399 \pm 45.5^{* * *}$ & $259 \pm 45.6^{* * *}$ & $140(-2.0-282.8)$ & 0.05 \\
\hline 6 months & $332 \pm 47.8^{* * *}$ & $307 \pm 47.6^{* * *}$ & $25(-123.4-171.9)$ & 0.75 \\
\hline \multicolumn{5}{|c|}{$\begin{array}{l}\text { COPD Assessment } \\
\text { Test score }\end{array}$} \\
\hline Baseline & $15.8 \pm 0.5$ & $15.2 \pm 0.6$ & & \\
\hline 2 months & $-3.5 \pm 0.6^{* *}$ & $-2.1 \pm 0.6 * *$ & $-1.4(-3.4-0.6)$ & 0.17 \\
\hline 6 months & $-3.6 \pm 0.6^{* *}$ & $-2.2 \pm 0.6 * *$ & $-1.4(-3.5-0.6)$ & 0.18 \\
\hline
\end{tabular}

gain of $38 \mathrm{~m}$ in the stable pulmonary rehabilitation group. This resulted in a difference of $33.9 \mathrm{~m}(95 \% \mathrm{CI}$ 4.2-63.7) $\mathrm{p}=0.02$, which is below the minimal clinical important difference (MCID) of $47.5 \mathrm{~m}$ [20]. In addition, there were not a significantly greater number of participants who gained $47.5 \mathrm{~m}$ in the early pulmonary rehabilitation group (responders) $(\mathrm{p}=0.07)$. After 6 months, the difference in ISWT between the two groups diminished to $17.7 \mathrm{~m}(95 \% \mathrm{CI}-13.3-48.7) \mathrm{p}=0.26$. There was a trend towards a larger improvement in ESWT in the early pulmonary rehabilitation group at 2 months (140.4 s (95\% CI $-2.0-282.8) \mathrm{p}=0.05)$ but no difference at 6 months $(24.3 \mathrm{~s}(95 \% \mathrm{CI}-123.4-171.9) \mathrm{p}=0.75)$. The MCID of 186-199 s was not reached [21].

Health-related quality of life measured by CAT was not significantly different at either 2 months $(-1.4$ point $(95 \% \mathrm{CI}-3.4-0.6) \mathrm{p}=0.17$ ) or 6 months $(-1.4$ point $(95 \% \mathrm{CI}-3.5-0.6) \mathrm{p}=0.18)$ (figure 5 ).

\section{Discussion}

This study demonstrated that initiating a standardised pulmonary rehabilitation programme shortly after hospitalisation due to severe AECOPD did not improve 1-year survival or reduce 1-year risk of hospital admissions compared to rehabilitation initiated during the later stable phase. Despite that, we found early pulmonary rehabilitation to have consistently greater benefit throughout the first 6 months of follow-up with regard to physical performance assessed by the shuttle walking tests, and additionally, the participants in the early pulmonary rehabilitation group had significantly higher adherence than those initiated their rehabilitation later. However, the differences between the groups regarding performance in the shuttle walking tests diminished after 6 months showing a nonsustained improvement.
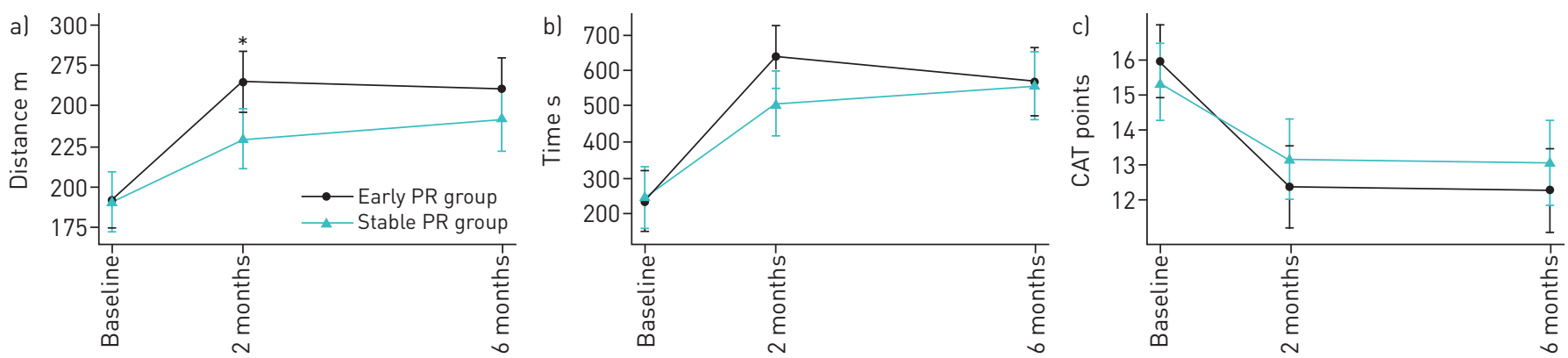

FIGURE 5 Exercise capacity and quality of life. Functional measures in al incremental shuttle walk test and b) endurance shuttle walk test, and cl quality of life in COPD Assessment Test at baseline, 2 months and 6 months follow-up. PR: pulmonary rehabilitation. *: $p<0.05$. 
In our study, the stable pulmonary rehabilitation group received the same rehabilitation as the early intervention group, only differing in timing. Only one study has previously compared early (within 2 weeks of an exacerbation) with late pulmonary rehabilitation (6 months after exacerbation) [22]. However, this study was underpowered due to recruiting issues, but the conclusion supported our results.

The result on primary outcome came out negative. However, the confidence intervals of the results were wide, suggesting limited confidence that the results confirm lack of effect. The sample size calculation was derived from the highly positive Cochrane review from 2011 on pulmonary rehabilitation in AECOPD, which today seems to have overestimated the effects. Estimated from our results, we would have had to include four times as many participants to see a significant effect of the intervention.

The updated Cochrane review from 2016, European Respiratory Society (ERS)/American Thoracic Society (ATS) guidelines on the management of COPD exacerbations and a systematic review by RYRS $\varnothing$ et al. $[23,24]$ concluded that early pulmonary rehabilitation implemented 3 weeks after discharge, reduced hospital admissions. But in contrast to these reviews, which compared early pulmonary rehabilitation to no rehabilitation, the present study compares early to late onset rehabilitation. Even though the readmission rate was high in our study, with 59\% readmitted due to all causes during the 1-year observation period and $46 \%$ due to respiratory causes, we did not find a significant reduction in the risk of either readmissions or exacerbations. Previous attempts to explain divergent findings in earlier trials included lack of comprehensiveness in the rehabilitation programme $[9,25]$. As our intervention fulfilled the criteria for a pulmonary rehabilitation programme according to general guidelines, we do not expect it as a likely explanation for our negative finding.

Further, we did not find reduced mortality, which aligns with the ERS/ATS guideline and Cochrane review [23]. The mortality in this study was notably low but this is mainly explained by the exclusion of patients who died in-hospital during admission for AECOPD and before inclusion in the present study.

With regard to exercise capacity, we found a significant improvement in incremental shuttle walk (ISWT) and borderline significance in endurance shuttle walk (ESWT) after 2 months in participants who recieved early pulmonary rehabilitation. However, both differences between groups are below the MCID [20, 21]. At 2 months, which was before initiation of stable rehabilitation, the stable pulmonary rehabilitation group did show a spontaneous large increase in exercise capacity in both ISWT and ESWT. An explanation for this could be natural recovery, which has been seen in previous studies, although not to the same extent $[9,26,27]$. The stable pulmonary rehabilitation group was not allowed to train elsewhere before the 2-month follow-up and seven participants in that group were excluded due to their participation in another rehabilitation programme. Further, the stable pulmonary rehabilitation group did unfortunately not gain the expected effects of the standard rehabilitation programme. This is probably explained by the low adherence to the rehabilitation programme. The early intervention group had an expected improvement in COPD assessment test, as seen in other trials but, as mentioned, we also found a large spontaneous recovery in the stable pulmonary rehabilitation group [28]. Surprisingly, we did not observe the expected worsening in quality of life after 6 months in the intervention group, as seen in other studies [14].

The current study was designed to emulate a regular clinical setting with patients referred to the same standard pulmonary rehabilitation programme as patients with stable COPD, so it would be feasible to implement in daily clinical practice. Uptake of early pulmonary rehabilitation was acceptable at $83 \%$ and the adherence was significantly higher in the early compared to the stable intervention group, which is probably explained by the participants' commitment to rehabilitation shortly after a hospitalisation. It was possible to refer patients to early pulmonary rehabilitation, but the challenge was to get them to attend the rehabilitation sessions, even though transportation to the rehabilitation centre was arranged for free.

A key limitation in the current study was the exclusion of eight participants during the study period, mainly due to misclassification of COPD (e.g. at follow-up, they presented a normalised spirometry or a substantial reversibility and thereby fulfilled criteria for asthma)

Another limitation was a similar recruiting problem as seen in other studies investigating the effect of rehabilitation in exacerbations of COPD $[22,29]$. Hospital admissions due to exacerbation of COPD in Denmark usually last 3-4 days, and as the patients were asked to participate within the first couple of days after admission; rehabilitation may have seemed overwhelming, which made some patients decline participation, as also seen in other trials $[30,31]$. This is a known problem when evaluating pulmonary rehabilitation in an acute setting and may results in a selection bias, making the included participants not representative of the general COPD population. The uptake of early rehabilitation needs to be investigated further especially in the most symptomatic patients (e.g. a referral visit within 1 week after discharge when the patients have recovered from the most severe part of the exacerbation). 


\section{Conclusion}

We found that early pulmonary rehabilitation after severe exacerbation of COPD seems to accelerate the recovery of exercise capacity, assessed as ISWT, compared to pulmonary rehabilitation initiated later in the stable phase. In addition, we found that early pulmonary rehabilitation was safe and associated with higher adherence to the rehabilitation programme. However, we did not find any effect on mortality or hospital readmission rate.

Acknowledgements: the authors would like to thank our research and rehabilitation team: M. Rosenberg, A. Behring, P. Kjerrumgaard, H. Rohde, L. Dalhof and H. Riber. In addition, we acknowledge the support of the Department of Respiratory Medicine, Gentofte Hospital, Denmark and the Prevention Fund of the Capital Region in Denmark.

Author contributions: J.L. Kjærgaard, C.B. Juhl and J.T. Wilcke contributed to the design of the study and conception and acquisition of data. J.L. Kjærgaard and C.B. Juhl analysed data, and J.T. Wilcke provided statistical advice. All authors contributed to the writing of the manuscript, interpreted and critically revised the work. They all approved the final version and are accountable for their part of the work.

Conflict of interest: None declared.

Support statement: This study was supported by the Prevention Fund of the Capital Region in Denmark. Funding information for this article has been deposited with the Crossref Funder Registry.

\section{References}

1 Dransfield MT, Kunisaki KM, Strand MJ, et al. Acute exacerbations and lung function loss in smokers with and without chronic obstructive pulmonary disease. Am J Respir Crit Care Med 2017; 195: 324-330.

2 Burrows B, Bloom JW, Traver GA, et al. The course and prognosis of different forms of chronic airways obstruction in a sample from the general population. N Engl J Med 1987; 317: 1309-1314.

3 Suissa S, Dell'Aniello S, Ernst P. Long-term natural history of chronic obstructive pulmonary disease: severe exacerbations and mortality. Thorax 2012; 67: 957-963.

4 Clini EM, Crisafulli E, Costi S, et al. Effects of early inpatient rehabilitation after acute exacerbation of COPD Respir Med 2009; 103: 1526-1531.

5 Kessler R, Ståhl E, Vogelmeier C, et al. Patient understanding, detection, and experience of COPD exacerbations: an observational, interview-based study. Chest 2006; 130: 133-142.

6 Seemungal TAR, Donaldso GC, Bhowmi A, et al. Time course and recovery of exacerbations in patients with chronic obstructive pulmonary disease. Am J Respir Crit Care Med 2000; 161: 1608-1613.

7 McCarthy B, Casey D, Devane D, et al. Pulmonary rehabilitation for chronic obstructive pulmonary disease Cochrane Database Syst Rev 2015; 2015: CD003793.

8 Moore E, Palmer T, Newson R, et al. Pulmonary rehabilitation as a mechanism to reduce hospitalizations for acute exacerbations of COPD: a systematic review and meta-analysis. Chest 2016; 150: 837-859.

9 Greening NJ, Williams JEA, Hussain SF, et al. An early rehabilitation intervention to enhance recovery during hospital admission for an exacerbation of chronic respiratory disease: randomised controlled trial. BMJ 2014; 349 : g4315.

10 Puhan MA, Scharplatz M, Troosters T, et al. Pulmonary rehabilitation following exacerbations of chronic obstructive pulmonary disease. Cochrane Database Syst Rev 2016; 2016: CD005305.

11 Puhan MA, Gimeno-Santos E, Scharplatz M, et al. Pulmonary rehabilitation following exacerbations of chronic obstructive pulmonary disease. Cochrane Database Syst Rev 2011; 2011: CD005305.

12 Singh SJ, Morgan MD, Scott S, et al. Development of a shuttle walking test of disability in patients with chronic airways obstruction. Thorax 1992; 47: 1019-1024.

13 Eaton T, Young P, Nicol K, et al. The endurance shuttle walking test: a responsive measure in pulmonary rehabilitation for COPD patients. Chron Respir Dis 2006; 3: 3-9.

14 Dodd JW, Marns PL, Clark AL, et al. The COPD assessment test (CAT): short- and medium-term response to pulmonary rehabilitation. COPD J Chronic Obstr Pulm Dis 2012; 9: 390-394.

15 Vestbo J, Hurd SS, Agustí AG, et al. Global strategy for the diagnosis, management, and prevention of chronic obstructive pulmonary disease GOLD executive summary. Am J Respir Crit Care Med 2013; 187: 347-365.

16 Lange P, Blands J, Skrubbeltrang C, et al. National Klinisk Retningslinje for Rehabilitering af Patienter med KOL. Sundhedstyrrelsen København, Sundhedsstyrelsen, 2014.

17 Bolton CE, Bevan-Smith EF, Blakey JD, et al. British Thoracic Society guideline on pulmonary rehabilitation in adults: accredited by NICE. Thorax 2013; 68: iil-ii30.

18 GOLD 2017. Global Strategy for the Diagnosis, Management and Prevention of Chronic Obstructive Pulmonary Disease 2017 Report. http://goldcopd.org/gold-2017-global-strategy-diagnosis-management-prevention-copd.

19 Eriksen N, Vestbo J. Management and survival of patients admitted with an exacerbation of COPD: comparison of two Danish patient cohorts. Clin Respir J 2010; 4: 208-214.

20 Singh SJ, Jones PW, Evans R, et al. Minimum clinically important improvement for the incremental shuttle walking test. Thorax 2008; 63: 775-777.

21 Altenburg WA, Duiverman ML, ten Hacken NHT, et al. Changes in the endurance shuttle walk test in COPD patients with chronic respiratory failure after pulmonary rehabilitation: the minimal important difference obtained with anchor- and distribution-based method. Respir Res 2015; 16: 27.

22 Puhan MA, Spaar A, Frey M, et al. Early versus late pulmonary rehabilitation in chronic obstructive pulmonary disease patients with acute exacerbations: a randomized trial. Respiration 2012; 83: 499-506.

23 Wedzicha JA, Miravitlles M, Hurst JR, et al. Management of COPD exacerbations: a European Respiratory Society/American Thoracic Society guideline. Eur Respir J 2017; 49: 1600791.

24 Ryrsø CK, Godtfredsen NS, Kofod LM, et al. Lower mortality after early supervised pulmonary rehabilitation following COPD-exacerbations: a systematic review and meta-analysis. BMC Pulm Med 2018; 18: 154. 
25 Ko FWS, Dai DLK, Ngai J, et al. Effect of early pulmonary rehabilitation on health care utilization and health status in patients hospitalized with acute exacerbations of COPD. Respirology 2011; 16: 617-624.

26 Seymour JM, Moore L, Jolley CJ, et al. Outpatient pulmonary rehabilitation following acute exacerbations of COPD. Thorax 2010; 65: 423-428.

27 Man WD-C, Polkey MI, Donaldson N, et al. Community pulmonary rehabilitation after hospitalisation for acute exacerbations of chronic obstructive pulmonary disease: randomised controlled study. BMJ 2004; 329: 1209.

28 Dodd JW, Hogg L, Nolan J, et al. The COPD assessment test (CAT): response to pulmonary rehabilitation. A multicentre, prospective study. Thorax 2011; 66: 425-429.

29 Ko FWS, Cheung NK, Rainer TH, et al. Comprehensive care programme for patients with chronic obstructive pulmonary disease: a randomised controlled trial. Thorax 2016; 72: 122-128.

30 Benzo R, Wetzstein $\mathrm{M}$, Neuenfeldt $\mathrm{P}$, et al. Implementation of physical activity programs after COPD hospitalizations: lessons from a randomized study. Chron Respir Dis 2015; 12: 5-10.

31 Jones SE, Green SA, Clark AL, et al. Pulmonary rehabilitation following hospitalisation for acute exacerbation of COPD: referrals, uptake and adherence. Thorax 2014; 69: 181-182. 